\title{
CALIDAD DE VIDA DEL ADULTO CON INSUFICIENCIA RENAL CRÓNICA, UNA MIRADA BIBLIOGRÁFICA
}

\section{ADULT’S LIFE QUALITY WITH CHRONIC KIDNEY DISEASE, A BIBLIOGRAPHIC VIEW}

\author{
TITULO CORTO: CALIDAD DE VIDA DEL ADULTO CON INSUFICIENCIA \\ RENAL CRÓNICA
}
Yadira Pabón-Varela ${ }^{1}$, Kemberly Saray Paez-Hernandez ${ }^{2}$, Kelly Dayanna Rodriguez-Daza ${ }^{2}$, Carolina Eustralia Medina-Atencia ${ }^{2}$, Melissa López-Tavera² ${ }^{2}$ Laura Vanessa Salcedo-Quintero ${ }^{2}$

Recibido en noviembre 28 de 2014

Aceptado en abril 27 de 2015

\section{RESUMEN}

El objetivo fue describir la calidad de vida de pacientes con disminución de la función renal. Como materiales y métodos se realizaron una revisión bibliográfica en las bases de datos (Pubmed, biblioteca virtual de salud, Scielo, Lilacs, Redalyc y Google académico), utilizando descriptores como: calidad de vida/relaciones enfermeropaciente/insuficiencia renal/enfermedad crónica. Se seleccionaron 52 artículos que midieran calidad de vida, de estos se excluyeron aquellas bibliografías con más de 6 años de antigüedad, los estudios que prevalecieron fueron los descriptivos. Los resultados arrojaron que la dimensión de calidad de vida más afectada es la física; de las tres modalidades de tratamiento para la insuficiencia renal, la que más afecta la calidad de vida del paciente es la hemodiálisis; el instrumento validado y más utilizado para medir calidad de vida en individuos con enfermedades crónicas es el SF-36. Como conclusiones: La calidad de vida de los pacientes con insuficiencia renal crónica se ve afectada, por lo que están sujetos a cambios en su estilo de vida que van de la mano con el tratamiento sustitutivo renal y sus complicaciones, además, presentan deterioro de la función física, mental, emocional, laboral y espiritual.

Palabras clave: Calidad de Vida; Insuficiencia Renal; Adulto. (Fuente: DeSC)

\section{AвSTRACT}

The objective was describe the quality of life of patients with decreased kidney function through a literature review in the various databases. As materials and methods a literature review was performed in the databases (Pubmed, virtual health library, Scielo, lilacs, Redalyc and Google scholar), using descriptors such as: quality of life/Relations

1. Enfermera de la Universidad del Norte. Magíster en enfermería para el cuidado de la salud cardiovascular de la Universidad Nacional de Colombia. Profesora investigadora del Programa de Enfermería de la Universidad Cooperativa de Colombia, sede Santa Marta-Colombia. Correo electrónico: yadira.pabon@ campusucc.edu.co.

2. Estudiantes de Enfermería de la Universidad Cooperativa de Colombia, Sede Bucaramanga-Colombia. Correos electrónicos: kesaph_1091@hotmail.com; dayanna10k@hotmail.com; carolina.medina.atencia@hotmail.com; meli_jm93@hotmail.com; vane.salcedo.quintero@gmail.com. 
nurse-patient/renal failure/chronic disease. We selected 52 articles whose studies measured the quality of life, excluding bibliography greater than 6 years of age; in which the prevailing type of descriptive study. The results showed that the dimension most affected is the physical, of the three modalities of treatment for kidney failure, which presents a greater involvement of the quality of life in the patient is hemodialysis, the validated instrument and more used to measure quality of life in individuals with chronic illnesses is the SF-36. As conclusions the quality of life of patients with chronic renal failure is affected, are subject to change in your lifestyle that go hand in hand with the renal replacement therapy and its complications, moreover, show deterioration in physical, mental, emotional, and spiritual labor.

Keywords: Quality of Life; Renal Insufficiency; Adult. (Fuente: DeSC)

\section{INTRODUCCIÓN}

A ctualmente, la población adulta se ve afectada por enfermedades crónicas como la insuficiencia renal crónica, la cual, en su fase avanzada toma el carácter de enfermedad terminal afectando de manera significativa la calidad de vida de quien la padece ${ }^{1}$. La insuficiencia renal crónica terminal (IRCT) es una enfermedad de pronóstico reservado, con desenlace mortal a corto o mediano plazo; sin embargo, los tratamientos actuales como la hemodiálisis, diálisis peritoneal y el trasplante renal han mejorado la sobrevida de estos pacientes ${ }^{2,3}$.

La prevalencia e incidencia de la enfermedad renal crónica avanzada, que requiere terapia de remplazo renal, ha crecido de manera progresiva en Colombia y en la mayoría de los países del mundo; en la actualidad hay aproximadamente 20.000 personas en terapia de remplazo renal en Colombia, lo que equivale a una prevalencia aproximada de 450 pacientes por millón de habitantes. Con una incidencia alrededor de $5 \%{ }^{4}$.

La insuficiencia renal crónica es la forma más grave y devastadora de enfermedad renal; puede llevar inexorablemente a la muerte del paciente si no se inicia un tratamiento oportuno y adecuado, es abordada por tres tratamientos que indiscutiblemente intervienen en la calidad de vida del paciente ${ }^{5,6}$, la diálisis peritoneal, procedimiento que se lleva por medio de la membrana interna del peritoneo, la cual recubre la zona abdominal, se encarga de filtrar la sangre removiendo el exceso de agua, desechos y sustancias químicas; ; la hemodiálisis que se realiza a través de una máquina que se encarga de purificar y filtrar temporalmente la sangre; para finalizar, el trasplante renal es un procedimiento de gran envergadura e involucra un cambio total del estado de salud y de la calidad de vida, es una transferencia de tejidos u órganos (riñón) desde un organismo donante a otro receptor ${ }^{8}$.

Según la OMS calidad de vida es "la percepción individual de la propia posición en la vida dentro del contexto del sistema cultural y de valores en que se vive y en relación con sus objetivos, esperanzas, normas y preocupaciones"9; la medida de la calidad de vida relacionada con la salud (CVRS), ayuda a determinar los efectos que produce la enfermedad en el paciente y la forma en la que afecta todos los aspectos de su vida, determinados por el proceso salud-enfermedad incluyendo su terapeutica ${ }^{10}$. La CVRS permite además establecer el bienestar físico que es determinado por la actividad funcional del individuo; el bienestar social tiene que ver con las funciones y las relaciones relacionadas con el trabajo, la situación económica y el sufrimiento familiar ${ }^{11}$, el bienestar psicológico y espiritual se relacionan con el temor, la ansiedad y la angustia que genera la enfermedad y el tratamiento, incluso comprende la religiosidad, la esperanza y la fortaleza interior ${ }^{12,13}$.

Para la realización de este trabajo se planteó como objetivo general describir de qué manera se ve afectada la calidad de vida en el adulto con insuficiencia renal crónica, mediante una revisión bibliográfica que permita identificar las metodologías que han sido utilizadas en los diferentes estudios; además, analizar el nivel de afectación en los diferentes ámbitos de desempeño y establecer cuáles intervenciones de enfermería contribuyen al mejoramiento de la vida de estos pacientes. 


\section{MATERIALES Y MÉTODOS}

Revisión bibliográfica profunda llevada a cabo mediante la selección de artículos de las diversas bases de datos (pubmed, biblioteca virtual de salud, Scielo, Lilacs, Redalyc y Google académico), con el fin de recopilar la mayor información verídica disponible referente al tema. Se realizó la búsqueda en las bases de datos utilizando descriptores como: calidad de vida/relaciones enfermeropaciente/insuficiencia renal/enfermedad crónica.

Se consultaron 70 artículos de investigación, de los cuales se seleccionaron 52, teniendo en cuenta los siguientes criterios de inclusión: estudios que midieran la calidad de vida del paciente con enfermedad crónica; investigaciones con sujetos de estudio cuyas facultades mentales se encontraran preservadas, en edades no inferiores a los 18 años; usuarios que se encontraran en terapia renal sustitutiva superior a los 3 meses y artículos escritos en español, portugués e inglés, puesto que son las lenguas que más aportan literatura acorde al tema. Se excluyó bibliografía con más de 6 años de antigüedad.

Respecto a los sujetos de estudio que presentaban los artículos seleccionados, el $100 \%$ perteneció a personas con edades superiores a los 18 años y en terapia renal sustitutiva no inferior a los tres meses.

\section{RESULTADOS}

De acuerdo a la Figura 1, de los artículos consultados el tipo de estudio prevalente es el descriptivo, ya que permite la recolección de información sin cambiar el entorno y expone los datos sin ningún tipo de manipulación.

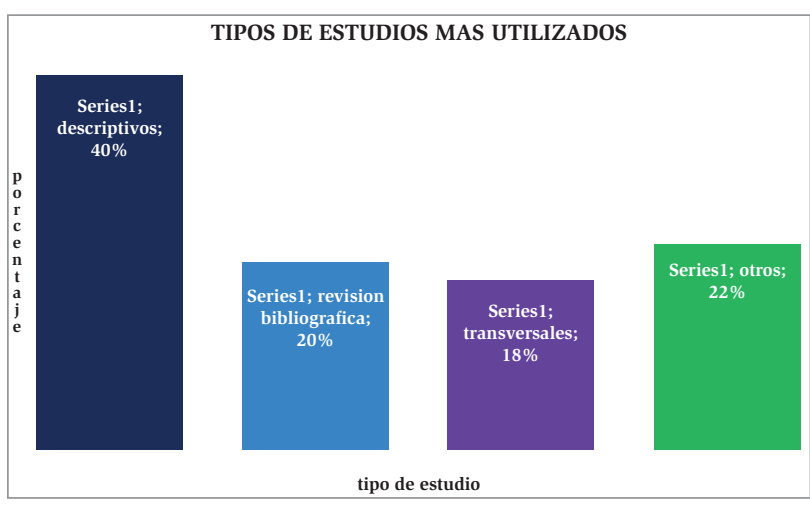

Figura 1. Tipo de estudios más utilizados
La dimensión de calidad de vida más afectada es la física debido a que el paciente pierde independencia en la realización de sus actividades diarias, además de someterse a múltiples tratamientos curativos altamente invasivos y demandantes ${ }^{14,15}$, los cuales deben acompañarse de una dieta estricta, toma de medicamentos y restricción de líquidos, esto conlleva a un deterioro considerable de la calidad de vida ${ }^{16}$ (Figura 2).

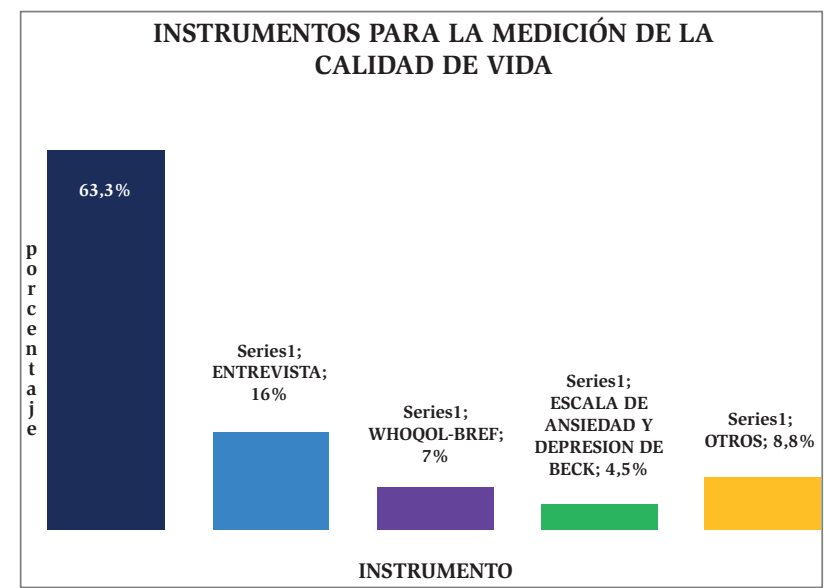

Figura 2. Dimensiones afectadas en el paciente.

De acuerdo a la Figura 3, el instrumento más utilizado para la medición de la calidad de vida en los pacientes con insuficiencia renal es el Kidney Disease Quality of Life (KDQOL-36; SF-36), debido a que valora de forma integral todos los aspectos que influyen en está ${ }^{17}$.

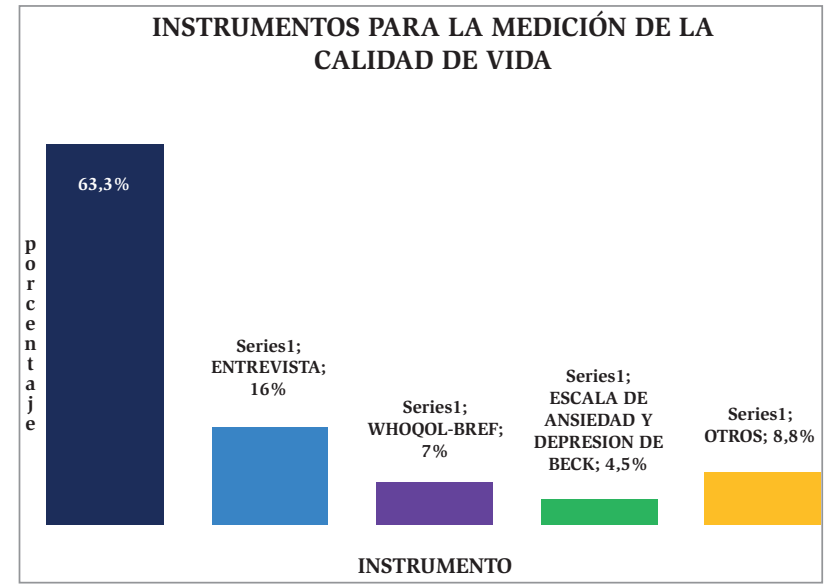

Figura 3. Instrumentos para la medición de calidad de vida 
Respecto al rol del profesional de enfermería en el paciente con insuficiencia renal crónica (IRC), se destaca como función primordial promover el autocuidado desde la educación, con la finalidad de contribuir a la construcción de la autonomía en la persona y su familia frente a su estado de salud, ya que los estudios demuestran que esto aporta significativamente al mejoramiento de la calidad de vida ${ }^{18}$.

La dimensión de salud mental en los pacientes con IRC, se ve afectada en la valoración realizada por el SF-36 ${ }^{19}$, porque estos en su mayoría presentan depresión durante el proceso de enfermedad y tratamiento ${ }^{20,21}$.

De las tres modalidades de tratamiento para la insuficiencia renal, la que presenta una mayor afectación de la calidad de vida en el paciente es la hemodiálisis ${ }^{22,23}$, ya que ésta requiere del traslado de tres o más veces por semana a la unidad renal ${ }^{24}$, viéndose así perjudicados los aspectos físicos, sociales, psicológicos y espirituales que conforman al individuo; por otra parte la diálisis peritoneal, aunque es de fácil manejo para éste, representa una mayor probabilidad de sufrir complicaciones, concluyendo así que el trasplante renal es la mejor opción entre los tratamientos sustitutivos de la función renal ${ }^{25}$, puesto que este mejora en grandes proporciones la condición de vida de las personas, representando de esta manera volver a la normalidad luego del padecimiento de la enfermedad renal crónica ${ }^{26,27}$.

\section{DISCUSIÓN}

Existe entre calidad de vida e IRC una estrecha relación toda vez que este tipo de insuficienciagenera impacto en la vida y en la funcionalidad física y mental de los pacientes.

Vinaccia et al. ${ }^{28}$, concluyen en su artículo que las personas con IRC confían en sus capacidades, son constantes, se adaptan a los cambios, se recuperan con facilidad de las crisis (competencia personal) y tienen aceptación tanto de sí mismos como de la vida (resiliencia); esto se logra gracias al proceso educativo brindado por el profesional de enfermería que permite el afianzamiento de la autonomía y el autocuidado en ellos.

Pérez menciona que muchos pacientes, ante el diagnóstico de insuficiencia renal crónica asumen una posición pesimista y fatalista hacia el futuro; de acuerdo a los hallazgos de la revisión, se evidencia que esto ocurre como consecuencia de la depresión por la que cursan estas personas durante su proceso de enfermedad ${ }^{29}$.
Segui et al. ${ }^{30} \mathrm{y}$ Carrillo et al. ${ }^{31}$ refieren que la pobre calidad de vida que tiene el paciente en tratamiento con diálisis, presenta una disminución representativa en todas las dimensiones del test de salud, y no solamente como cabría de esperar, en afectación del estado físico; esto como consecuencia a los requerimientos de esta terapia, aunque cabe resaltar, que dentro de todas las posibilidades de tratamiento es la que menos repercusiones tiene y que en menor proporción afecta la dimensión física, laboral y social ${ }^{22-34}$.

Capote et al..$^{35} \mathrm{y}$ Varela et al. ${ }^{36}$ exponen que el tratamiento con hemodiálisis genera en los pacientes inseguridad con respecto al futuro, la dependencia de máquinas y personal sanitario, las restricciones en la dieta, los cambios en el rol social y familiar, en el aspecto físico y en la situación laboral; acorde a lo afirmado por estos autores la hemodiálisis se convierte en la terapia renal sustitutiva que más influye negativamente en la calidad de vida de las mujeres y hombres que padecen IRC ${ }^{37}$.

Assis et al. ${ }^{38} \mathrm{y}$ Cruz et al. ${ }^{39}$, describen en su estudio que solo el componente físico independiente muestra una correlación negativa con el aumento de la edad y de asociación femenina. Se concluyó que algunos factores como el aumento de la edad, el sexo femenino, y el número de hospitalizaciones se asociaron a una peor puntuación en la calidad de vida, mientras que Urzua et al. ${ }^{40,41}$, proponen que en cuanto a la calidad de vida relacionada con la salud no hay evidencias de diferencias estadísticamente significativas entre las medias de los hombres y mujeres, exceptuando la dimensión síntomas, donde la media de las mujeres es menor que la de los hombres.

\section{CONCLUSIONES}

La calidad de vida de las personas que padecen insuficiencia renal crónica se ve afectada debido a que están sujetos a cambios en su estilo de vida que van de la mano con el tratamiento sustitutivo renal y sus complicaciones; secundario a esto presentan un deterioro de la función física, mental, emocional, laboral y espiritual ${ }^{42,43}$.

De los 52 artículos seleccionados, el $40 \%$ corresponden a estudios de tipo descriptivo, revisiones bibliográficas con un porcentaje del $20 \%$, estudios de tipo transversal con un $18 \%$ y por último en menor cantidad estudios de tipo cohorte, investigación clínica, ex-post-factor y retrospectivo, que corresponden al $22 \%$. 
Entre los instrumentos que se utilizaron para medir la calidad de vida en los pacientes con enfermedad renal crónica, se encontró en una mayor aplicabilidad el Kidney Disease Quality of Life (KDQOL - 36; SF-36) con un $63.6 \%$, seguido por: la entrevista con un $16 \%$, la prueba The World Health Organization Quality of Life (WHOQOL)-BREF con un 7\%, la escala de ansiedad y la escala de depresión de Beck con un $4.5 \%$,y por ultimo con una sumatoria del $8.8 \%$ (ficha sociodemográfica, escala psicosocial del cuidador, la escala de carga de Zarit, inventario de ansiedad, y test de Morisky-Gree), con base en esto se concluye que el instrumento más apto para la medición de la calidad de vida implementado y validado en Colombia es el SF-36.

Es un objetivo prioritario de la consulta de enfermería renal crónica conocer qué tanta información sobre los diferentes tratamientos sustitutivos posee el paciente, de igual forma si este conoce sus derechos sobre la elección libre de su tratamiento y que tan informado esta sobre los cuidados especiales con los dispositivos y fistulas para el tratamiento renal sustitutivo (TRS), permitiendo con esto la disminución de la morbimortalidad y un mejoramiento de la calidad de vida del paciente ${ }^{44,45}$.

Es posible desarrollar un programa educativo, trabajando interdisciplinariamente entre enfermeras y médicos internistas que ayudará a detectar a aquellas personas que a mediano plazo requieran diálisis ${ }^{46-48}$. Así, con anticipación podrían empezar a realizar cambios en su estilo de vida para alargar el proceso de ingreso al programa de tratamiento, por ende tener conocimientos previamente de aquellos cambios a los que deberán irse adaptando y así podrían aceptar mejor la enfermedad y pensar en opciones una vez que llegue el momento de tomar la decisión, es decir tener un paciente mejor preparado para el futuro ${ }^{48,49}$.

El cuidado del paciente conlleva una gran carga y afectaciones en la esfera social, psicológica, económica, laboral y del estado de salud en general que pudiera repercutir en la calidad de vida del enfermo y del propio cuidador $^{50,51}$.

Se considera que dentro de la terapia renal sustitutiva, la diálisis peritoneal es aquella que afecta en menor magnitud la calidad de vida del individuo, porque permite un mejor desarrollo de su cotidianidad desde la perspectiva de la bioética en comparación con la hemodiálisis ${ }^{52}$.
Para finalizar se puede concluir que la calidad de vida está directamente relacionada con la salud en general, la interacción social, familiar y por último de la percepción propia respecto a la vida.

\section{REFERENCIASBIBLIOGRAFICAS}

1. Malheiro P, Arruda D. Percepciones de las personas con insuficiencia renal crónica sobre la calidad de vida. Rev. Enfermería global. 2012; 11 (4): 276 - 294.

2. Guerra V, Sanhueza O, Cáceres M. Calidad de vida de personas en hemodiálisis crónica: relación con variables sociodemográficas, médico-clínicas y de laboratorio. Revista. Latino-Am. Enfermagem. 2012 Oct; 20 (5): 838-846.

3. Yepes M, Montoya B, Orrego M, Cuéllar J, Yepes J, López $M$, et al. Calidad de vida en pacientes con enfermedad renal crónica sin diálisis ni trasplante de una muestra aleatoria de dos aseguradoras en salud. Medellín, Colombia, 2008; 29(6): 548- 556.

4. Gamarra G. Epidemiología de la insuficiencia renal crónica. Acta Med Colomb. 2013 jul; 38(3):116-117.

5. Rivera A, Montero L, Sandoval R. Desajuste psicológico, calidad de vida y afrontamiento en pacientes diabéticos con insuficiencia renal crónica en diálisis peritoneal. Salud Ment. 2012 Ago; 35(4): 329-337.

6. Cunha L, Teles L, Vasconcelos P, Alves M., Santana dos Santos J, De Oliveira R. Evaluación de la calidad de vida de pacientes de insuficiencia renal crónica en diálisis renal. Enferm. glob. 2011 Jul; 10(23): 158-164

7. Varela L, Vázquez M, Bolaños L, Alonso R. Predictores psicológicos de la calidad de vida relacionada con la salud en pacientes en tratamiento de diálisis peritoneal. Nefrología (Madr). 2011; 31(1): 97-106

8. Acosta A, Chaparro L, Rey C. Calidad de vida y estrategias de afrontamiento en pacientes con insuficiencia renal crónica sometidos a hemodiálisis, diálisis peritoneal o trasplante renal. Revista Colombiana de Psicología Universidad Nacional de Colombia 2008; 17 (1): 9-26.

9. Organización Mundial de la Salud. Programa Envejecimiento y Ciclo Vital. Envejecimiento activo: un marco político. Rev Esp Geriatr Gerontol 2002; 37(S2):74-105.

10. Vinaccia S, Quiceno J. Calidad de vida relacionada con la salud y enfermedad crónica: estudios colombianos. Psychol. av. discip. 2012; 6(1): 123-136.

11. Aparicio C, Fernández A, Garrido G, Luque-de Pablos A, Izquierdo E. Influencia de la situación clínica en la calidad de vida relacionada con la salud de los pacientes pediátricos con enfermedad renal crónica. Nefrología (Madr). 2013; 33 (1): 61-69 
12. Julián J, Molinuevo J, Sánchez J. La situación laboral del paciente con enfermedad renal crónica en función del tratamiento sustitutivo renal. Nefrología (Madr.) . 2012; 32(4): 439-445

13. Medellín J. Carrillo G. Soporte social percibido y calidad de vida de personas con enfermedad renal crónica sometidas a trasplante renal. Revista Avances en Enfermeria. 2014; 32 (2): 206 - 216.

14. Páez A, Jofré M, Azpiroz C, Bortoli M. Ansiedad y depresión en pacientes con insuficiencia renal crónica en tratamiento de diálisis. Universitas Psychologica. 2009; 8 (1): 117-124

15. Polo R, Meseguer S, Pérez M, Molina M, López M, Moreno J. ¿Cómo incide la actividad física en la satisfacción con la vida de los pacientes renales sometidos a hemodiálisis? Enfermeria Nefrologica. 2013; 16 (1): $156-157$

16. Malheiro P, Arruda D. Percepciones de las personas con insuficiencia renal crónica sobre la calidad de vida. Enferm. Glob. 2012 Oct; 11(28): 257-275

17. Chaves K, Duarte A, Vesga J. Adaptación transcultural del cuestionario KDQOL SF 36 para evaluar calidad de vida en pacientes con enfermedad renal crónica en Colombia. Rev.Fac.Med. 2013 Dic; 21(2): 34-42.

18. Contreras F, Espinosa J, Esguerra G. Calidad de vida, autoeficacia, estrategias de afrontamiento y adhesión al tratamiento en pacientes con insuficiencia renal crónica sometidos a hemodiálisis. Psicysalud. 2008; 118(02): 165-179.

19. Rodríguez M, Merino M, Castro M. Valoración psicométrica de los componentes físicos (csf) y mentales (csm) del sf-36 en pacientes insuficientes renales crónicos en tratamiento con hemodiálisis. Cienc. enferm. 2009 Abr]; 15(1 ): 75-88.

20. Hecmy G, Nicolina C. Calidad de vida percibida, depresión y ansiedad en pacientes con tratamiento sustitutivo de la función renal. Red de Revistas Científicas de América Latina y el Caribe, España y Portugal. 2008; 18 (01): 5-15.

21. Morales R, Salazar E, Flores F, Bochicchio T, López A. Calidad de vida relacionada con la salud en los pacientes con tratamiento sustitutivo renal: el papel de la depresión. GacMédMéx. 2008; 144(2): 91-97.

22. Takemoto A, Okubo P, Bedendo J, Carreira L. Avaliação da qualidad de vida hemidosos submetidosao tratamento hemodialítico. Rev. GaúchaEnferm. 2011 Jun; 32(2): 256-262

23. Kusumoto L, Marques S, Haas R. Adults and elderly on hemodialysis evaluation of health related quality of life. Acta paul. enferm. 2008; 21(spe): 152-159.

24. Guerra V, Díaz A, Vidal K. La educación como estrategia para mejorar la adherencia de los pacientes en terapia dialítica. Rev Cubana de Enfermeria. 2010; 26(2): 52 -62.
25. Prieto M, Escudero M, Suess A, March J, Ruiz A, Danet A. Análisis de calidad percibida y expectativas de pacientes en el proceso asistencial de diálisis. Anales Sis San Navarra. 2011 Abr; 34(1): 21-31.

26. Rodríguez M, Conejos C, Fuenmayor A, Mirada C, Sanz E, Torruella $\mathrm{C}$, et al. Calidad de vida y trasplante renal en mayores de 65 años. Rev Soc Esp Enferm Nefrol. 2009 Mar; 12(1): 26-30.

27. Borroto G, Almeida J, Lorenzo A, Alfonso S, Guerrero C. Percepción de la calidad de vida por enfermos sometidos a tratamientos de hemodiálisis o trasplante renal: Estudio comparativo. Rev cubana de Medicina. 2007; 46(3): 43 -50.

28. Vinaccia S, Quiceno J. Resiliencia y Calidad de Vida Relacionada con la Salud en Pacientes con Insuficiencia Renal Crónica - IRC. Revista Argentina de Clínica Psicológica 2011; 20 (3): 201 - 211.

29. Pérez G. Factores psicosociales y calidad de vida en pacientes con insuficiencia renal crónica. Revista de Psicología. 2010; 6: 69-76.

30. Seguí A, Amador P, Ramos A. Calidad de vida en pacientes con insuficiencia renal crónica en tratamiento con diálisis. Sociedad Española Enfermeria Nefrologica. 2010; 13 (3):155 - 160.

31. Carrillo A, Díaz F. Capacidad de autocuidado de los pacientes en diálisis peritoneal: un estudio piloto en Bogotá. Enferm. glob. 2013 Abr; 12(30): 54-64.

32. Zúñiga C, Dapueto J, Müller H, Kirsten L, Alid R, Ortiz L. Evaluación de la calidad de vida en pacientes en hemodiálisis crónica mediante el cuestionario "Kidney Disease Quality of Life (KDQOL-36)”. Rev. méd. Chile. 2009 Feb; 137(2): 200-207.

33. Oliveros M, Avendaño M, Bunout D, Hirsch S, De La Maza M, Pedreros C et al. Estudio piloto sobre entrenamiento físico durante hemodiálisis. Rev. méd. Chile. 2011 Ago; 139(8): 1046-1053.

34. Durán M, Lope A, Pino M, Chicharro M, Matilla E. Percepción de la calidad de vida referida por el paciente adulto con trasplante renal. EnfermNefrol. 2014 Mar; 17(1): 45-50.

35. Capote E, Casamayor Z, Castañer J. Calidad de vida y depresión en el adulto mayor con tratamiento sustitutivo de la función renal. RevCubMed Mil. 2012 Sep; 41(3): 237-247.

36. Varela D, Guarín M, Hincapié N, Hincapié S, Rodríguez K. Factores no tradicionales influyentes en la calidad de vida de los pacientes de hemodiálisis. Rev. Colomb. Nefrol. 2014; 1(1):17-24.

37. González R, Gómez C, Oviedo G, Villegas F. Calidad de vida relacionada con la salud y prevalencia de síntomas depresivos y ansiosos en pacientes en hemodiálisis, en seis unidades renales de bogotá, colombia. Rev. Colomb. Psiquiat. 2009; 38 (4): 622-636. 
38. Assis E, Gurgel M. Fatores asociados com a qualidade de vida relacionada à saúde de idososemhemodiálise. Rev. SaúdePública. 2011 Dec; 45(6): 1127-1136.

39. Cruz L, Fleck M, Oliveira M, Camey A, Hoffmann J, Bagattini Â, et al. Health-related quality of life in Brazil: normative data for the SF-36 in a general population sample in the south of the country. Ciênc. Saúde coletiva. 2013 Jul; 18(7): 1911-1921.

40. Urzúa A, Pavlov R, Cortés R, Pino V. Factores Psicosociales Relacionados con la Calidad de Vida en Salud en Pacientes Hemodializados. Terapia Psicológica 2011; 29 (1): 135-140.

41. Vinaccia S, Quiceno J. Calidad de vida relacionada con la salud y factores psicológicos: un estudio desde la enfermedad pulmonar obstructiva crónica - epoc. Ter Psicol. 2011 julio 1; 29(1): 65-75.

42. Pulido F, Arribas P, Fernández A. Satisfacción de los pacientes en la consulta de enfermería de enfermedad renal crónica avanzada. Revista de la Sociedad Española de Enfermeria Nefrologica. 2008 Sep; 11(3): 167-172.

43. Aguilera A, Prieto M, González L, Abad B, Martínez E, Robles I et al. Una estrategia poco utilizada en el cuidado de pacientes con enfermedad renal crónica: la educación en grupo y multidisciplinar de pacientes y sus familiares. Enferm Nefrol. 2012 Mar; 15(1): 14-21

44. Alarcón I, Farías D, Barrios S. Factores que influyen en la percepción de la calidad de vida de personas adultas bajo terapias de sustitución renal. Horizonte de enfermería. 2009; 20, (1): 67 - 80.
45. Sampaio C, Guedes M. Nursing process as a strategy in the development of competence for self-care. Actapaul. enferm. . 2012; 25(S2): 96-103.

46. Carrillo A, Laza C, Molina J. Estudio documental (20062013) sobre el autocuidado en el día a día del paciente con enfermedad renal crónica. EnfermNefrol. 2013 Sep; 16(3): 185-192.

47. Torres C. Diseño, implementación y evaluación de un programa de Intervención Educativa para pacientes con insuficiencia renal Crónica. Revista Latinoamericana de Medicina Conductual / Latin American Journal of Behavioral Medicine 2010; 1 (1): 37-46.

48. Leiva J, Sánchez R, García H, Fernández M, Heras M, Molina Á et al. Cuidados de soporte renal y cuidados paliativos renales: revisión y propuesta en terapia renal sustitutiva. Nefrología (Madr.) 2012; 32(1): 20-27.

49. Velázquez Y, Espín A. Repercusión psicosocial y carga en el cuidador informal de personas con insuficiencia renal crónica terminal. Revista Cubana de Salud Pública 2014; 40 (1): 3 - 17

50. Vargas L. Aporte de enfermería a la calidad de vida del cuidador familiar del paciente con alzheimer. Aquichan; 2012; 12(1): 62-76.

51. Torralba M, Pérez I. La calidad de vida del paciente nefrológico desde la perspectiva bioética. Enferm. glob. 2011 Oct; 10(24):210-217

52. Cardona D, Agudelo H. Construcción cultural del concepto calidad de vida. Revista Facultad Nacional de Salud Pública. 2005; 23(1):79-90.

Para citar este artículo: Pabón-Varela Y, Paez-Hernandez K, Rodriguez-Daza D, Medina-Atencia C, López-Tavera M, Salcedo-Quintero L. Calidad de vida del adulto con insuficiencia renal crónica, una mirada bibliográfica. Duazary. 2015 dic; 12 (2): 157 - 163 